\section{COVID-19 in a Young Girl with Restrictive Cardiomyopathy and Chronic Lung Disease}

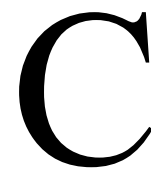

oronavirus disease (COVID-19) pandemic has resulted in severe infection with thousands of deaths among the elderly; however, very few pediatric cases need hospitalization and/or intensive care.

A 7-year-old female (weight $17 \mathrm{~kg}$ ), who was under our follow-up for the last one year with a diagnosis of cardiomyopathy and chronic lung disease (CLD), was admitted to the emergency unit with the complaints of chest pain, dyspnea and fatigue. She had no fever, cough, vomiting, myalgia or gastrointestinal symptoms. There was no exposure to a person infected with COVID-19 in her family. The body temperature was $36.5^{\circ} \mathrm{C}$, heart rate was 110 beats/minute, respiration rate was $24 /$ minute and blood pressure was $90 / 60 \mathrm{~mm} / \mathrm{Hg}$ on admission. She had bilateral rales, more prominent on the right side, and grade II systolic murmur on auscultation. The liver was 3$4 \mathrm{~cm}$ palpable below the right lower costal margin. She had no peripheric edema and her cutaneous oxygen saturation was $85 \%$. The chest $X$-ray showed infiltrations on the right middle and lower pulmonary zones and massive cardiomegaly (Fig. 1). Electrocardiogram showed sinus tachycardia and tall and wide $\mathrm{P}$ waves, suggesting bi-atrial dilatation. Laboratory results were normal except for raised white blood cell count (15500/ $\mathrm{mm}^{3}$ with $80 \%$ neutrophils) and blood urea of $55 \mathrm{mg} / \mathrm{dL}$. D-dimer and troponin levels were slightly increased ( 0.85 $\mathrm{mg} / \mathrm{L}$ and $0.3 \mathrm{ng} / \mathrm{mL}$, respectively). The erytrocyte sedimantation rate and $\mathrm{C}$-reactive protein levels were normal.

Echocardiography demonstrated restrictive cardiomyopathy, mitral and tricuspid insufficiency and left ventricular dysfunction (ejection fraction of $40 \%$ ). She had a history of using nasal continuous positive airway pressure (CPAP) and oxygen concentrator due to the CLD and oxygen desaturations $\left(\mathrm{SpO}_{2}: 80-85 \%\right)$. Her computed tomography (CT) of the chest, performed two months ago, was compatible with the pulmonary infiltration and CLD in the right lower and middle lobes. The patient was hospitalized and treatment started with inotropic support (milrinone, dopamine, furosemide infusion) and nasal CPAP. Her fever spikes increased further and CRP and neutrophil counts were also increased on the second day of admission. After the blood, sputum and urine cultures were obtained and the respiratory pathogen panel (RPP) was sent to the laboratory, antibiotic therapy was initiated with vancomycin and meropenem.

As COVID-19 infection was suspected due to her progressive deterioration, a nasopharyngeal swab test was obtained. The RPP and culture results were negative. The patient showed rapid deterioration within a few hours on the third day. Due to the acidosis, high lactate levels and hypercarbia on her blood gase analysis, she was intubated and ventilated, but she had a cardiac arrest. There was no response to the cardiopulmonary resuscitation. The swab test result was positive for COVID-19.

The common symptoms of COVID-19 reported in pediatric patients can easily be misdiagnosed as the upper/ lower respiratory tract infections of pediatric age group $[1,2]$. In a series of 2135 children from China, 728 of which were positive for COVID-19, 55\% were mild or asymptomatic, $40 \%$ were moderate (clinical or radiographic evidence of pneumonia without hypoxemia), and 5\% were severe (dyspnea, central cyanosis) and $<1 \%$ were critical [3]. Diagnosis of COVID-19 have been based on the presence of at least two symptoms (fever, cough, respiratory or gastrointestinal findings or fatigue), combined with laboratory tests (normal or low wbc count, low absolute lymphocyte count and increased CRP) and an abnormal chest $X$-ray [4]. The most commonly reported

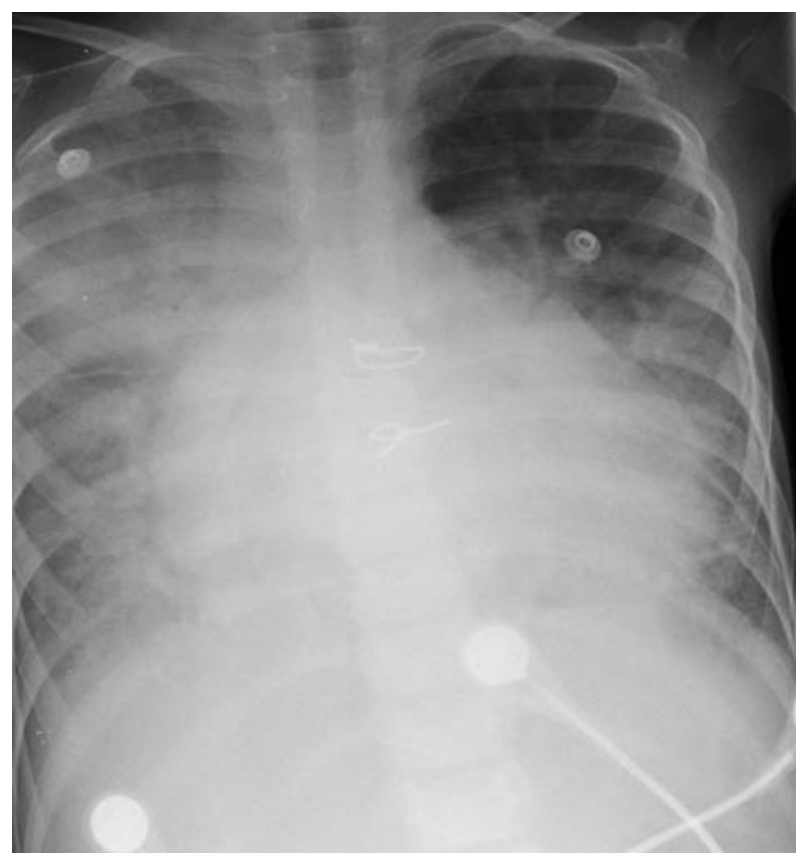

Fig. 1 Chest X-ray findings in a girl with COVID-19 infection and pre-existing restrictive cardiomyopathy and chronic lung disease. 
radiologic finding is bilateral ground-glass opacity (32.7\%) [5]. However, unlike adults, there are no typical radiological findings for definitive diagnosis of COVID19 in children.

In one series, $23 \%$ of 345 children with laboratoryconfirmed COVID-19 one or more comorbidity. The most commonly reported comorbid conditions were chronic pulmonary disease (including moderate to severe asthma), cardiovascular disease and immunosuppression [6]. Lu, et al. [5] reported that 3 out of 171 pediatric patients with COVID-19 required intensive care support and invasive mechanical ventilation; all with some underlying conditions, and only a 10-month-old child with intussuception died after 4 weeks of hospitalization [5].

Our case had no fever, no exposure to an infected person, but had restrictive cardiomyopathy and chronic lung disease that would suggest an exacerbation of her disease on admission. COVID-19 infection was suspected due to her progressive deterioration. We want to draw attention to such sub-acute clinical presentations of COVID-19 in the pediatric population with underlying comorbidities.

Published online: April 30, 2020; PII: S09745591600170

Contributors: AY: conception, design, drafting the manuscript; ATK: final drafting and preparing the version of the manuscript to be published.

Funding: None; Competing interest: None stated.
AYSE INCI YILDIRIM ${ }^{1 *}$ AND AYSU TURKMEN KARAAGAC ${ }^{2}$ Departments of ${ }^{1}$ Pediatric Cardiology and ${ }^{2}$ Pediatry, University of Health Sciences Kartal Kosuyolu Research and Training Hospital, Kartal/Istanbul.

\section{REFERENCES}

*ayildirimmd@yahoo.com

1. Wang D, Hu B, Hu C, Zhu F, Liu X, Zhang J, et al. Clinical characteristics of 138 hospitalized patients with 2019 novel coronavirus infected pneumonia in Wuhan, China. JAMA. 2020; 323:1061-9.

2. Qiu H, Wu J, Liang H, Yunling L, Song Q, Chen D. Clinical and epidemiological features of 36 children with coronavirus disease 2019 (COVID-19) in Zhejiang, China: An observational cohort study. Lancet Infect Dis. 2020. Available from: https://doi.org/10.1016/\$1473-3099 (20)30198-5. Accessed April 22, 2020.

3. Dong $\mathrm{Y}$, Mo X, Hu Y, Qi X, Jiang F, Jiang Z, et al. Epidemiology of COVID-19 among children in China. Pediatrics. 2020;145:e20200702.

4. Ludvigsson JF. Systematic review of COVID-19 in children shows milder cases and a beter prognosis than adults. Acta Paediatrica. 2020. Available from: https:// doi.org/10.1111/apa.15270. Accessed April 22, 2020.

5. Lu X, Zhang L, Du H, Zhang J, Li YY, Qu J, et al. SARSCoV-2 infection in children. $\mathrm{N}$ Engl J Med. 2020;382:1663-5.

6. Bialek S, Gierke R, Hughes M, McNamara LA, Pilishvili T, Skoff T. Coronavirus disease 2019 in children- United States, February 12-April 2, 2020. MMWR Morb Mortal Wkly Rep. 2020;69:422-6. 